Tecno Lógicas

ISSN 0123-7799

Vol. 18, No. 34, pp. 13-24

Enero-junio de 2015

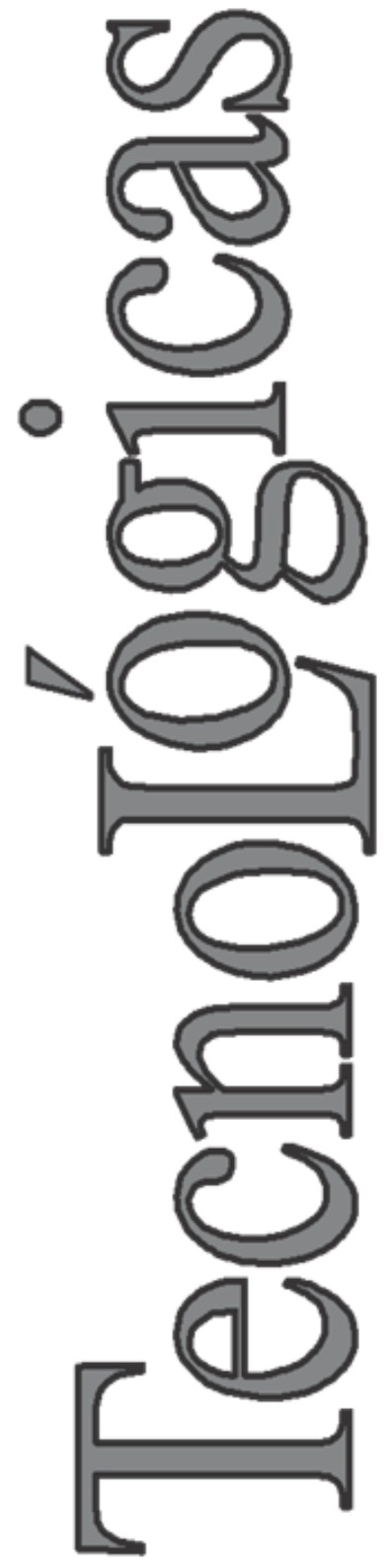

C) Copyright 2015 por autores y Tecno Lógicas Este trabajo está licenciado bajo una Licencia Internacional Creative Commons Atribución (CC BY)

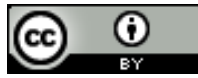

\section{Control GPI-repetitivo para sistemas lineales con incertidumbre/variación en los parámetros}

\section{GPI-repetitive control for linear systems with parameter uncertainty / variation}

\author{
John A. Cortés-Romero ${ }^{1} \mathrm{y}$ \\ Germán A. Ramos-Fuentes ${ }^{2}$
}

Recibido: 09 de marzo de 2014, Aceptado: 15 de julio de 2014

Cómo citar / How to cite

J. A. Cortés-Romero y G. A. Ramos-Fuentes, "Control GPI-repetitivo para sistemas lineales con incertidumbre/variación en los parámetros", Tecno Lógicas, vol. 18, no. 34, pp. 13-24, 2015.

1 Doctor en Ingeniería Eléctrica, Departamento de Ingeniería Eléctrica y Electrónica, Facultad de Ingeniería, Universidad Nacional de Colombia, Bogotá-Colombia, jacortesr@unal.edu.co

2 Doctor en Ingeniería Eléctrica, Departamento de Ingeniería Eléctrica y Electrónica, Facultad de Ingeniería, Universidad Nacional de Colombia, Bogotá-Colombia, garamosf@unal.edu.co 


\section{Resumen}

El problema de control de sistemas lineales para seguimiento o rechazo de señales periódicas con incertidumbre / variación paramétrica ha sido considerado desde varias perspectivas. Este trabajo presenta una propuesta de combinación de la técnica de control repetitivo con la técnica de control Proporcional Integral Generalizado (GPI). La adecuación y combinación de estas técnicas se realiza en un contexto de tiempo discreto. El control repetitivo se presenta como una técnica, basada en el principio del modelo interno, apta para el rechazo o seguimiento de señales periódicas. Por otra parte, la técnica de control GPI es una estrategia de diseño de controladores robustos lineales capaces de gestionar adecuadamente tanto perturbaciones exógenas de tipo estructurado así como la incertidumbre / variación de planta. Se plantea que el control GPI garantice unas condiciones de estabilidad y desempeño adecuadas para el correcto funcionamiento del control repetitivo sobre plantas lineales que refieran incertidumbre / variación paramétrica. Se presentan adicionalmente análisis de estabilidad y desempeño en términos de la respuesta en frecuencia tomando diferentes escenarios de incertidumbre en los parámetros y graficando las condiciones de estabilidad. Complementariamente, se realizan comparaciones con estrategias clásicas a nivel del control complementario, que han resultado efectivas para problemas de control similares, y se ilustran los análisis por medio de simulaciones.

\section{Palabras clave}

Control repetitivo, control GPI, control robusto, variación de parámetros, incertidumbre en los parámetros.

\section{Abstract}

Robust repetitive control problems for uncertain linear systems have been considered by different approaches. This article proposes the use of Repetitive Control and Generalized Proportional Integral (GPI) Control in a complementary fashion. The conditioning and coupling of these techniques has been done in a time discrete context. Repetitive control is a control technique, based on the internal model principle, which yields perfect asymptotic tracking and rejection of periodic signals. On the other hand, GPI control is established as a robust linear control system design technique that is able to reject structured time polynomial additive perturbation, in particular, parameter uncertainty that can be locally approximated by time polynomial signal. GPI control provides a suitable stability and robustness conditions for the proper Repetitive Control operation. A stability analysis is presented under the frequency response framework using plant samples for different parameter uncertainty conditions. We carry out some comparative stability analysis with other complementary control approaches that has been effective for this kind of task, enhancing a better robustness and an improved performance for the GPI case. Illustrative simulation examples are presented which validate the proposed approach.

\section{Keywords}

Repetitive control, GPI control, robust control, time varying parameters, uncertain parameters. 


\section{INTRODUCCIÓN}

En el diseño de sistemas de control, los cambios en la planta pueden ser modelados como incertidumbres o variaciones paramétricas y pueden ser adecuadamente tratados por medio de una estrategia de control que proporcione un grado de robustez suficiente para garantizar la estabilidad.

El control repetitivo es una técnica basada en el principio del modelo interno que ha sido muy eficiente en aplicaciones de control que requieran el seguimiento o rechazo de señales periódicas [1]-[6], una de sus principales debilidades es la necesidad de compensar el comportamiento dinámico de la planta. Ello hace que pueda presentar problemas de robustez en el caso de plantas inciertas. El problema de la compensación dinámica de la planta se ha trabajado desde diferentes contextos incluyendo estrategias de control adaptable [7], [8] y técnicas de control robusto principalmente [9]-[11].

El diseño del control repetitivo considera el modelo nominal de la planta; cualquier desviación de este modelo causada por incertidumbre en la planta o dinámicas no modeladas puede originar degradación del desempeño o incluso inestabilidad del sistema de control. Para compensar este problema se propone utilizar una filosofía de diseño basada en el rechazo activo de perturbaciones [12], bajo la técnica del controlador GPI [13], como control complementario al repetitivo con el objetivo de proveer al sistema la robustez necesaria.

Se pretende con la estrategia de control robusto GPI subyacente proveer una robustez, desde el control repetitivo, ante los cambios ocasionados en la planta por la variación de parámetros de tal forma que se garantice un alto desempeño del control repetitivo bajo situaciones adversas de incertidumbre/variación de la planta.
Ya se reportan resultados experimentales de la utilización de la técnica GPI para el manejo de dinámicas no modeladas e incertidumbre en los parámetros con excelentes resultados en su desempeño [14][17], que dieron motivación a esta investigación y garantía de los resultados esperados con este trabajo. Este trabajo forma parte de una serie de trabajos, aun preliminares, que buscan explorar capacidades y funcionalidades de la combinación de ambas técnicas con el fin de alcanzar sistemas de control que sean capaces de gestionar adecuadamente la incertidumbre/variación de planta.

En este artículo se presenta una metodología de diseño que involucra la estrategia de control repetitivo soportada sobre la base de una estrategia de control robusto implementada por medio de controladores GPI. Se analizan diferentes condiciones de estabilidad presentando casos extremos y se comparan con diferentes estrategias clásicas adoptadas para el controlador complementario. En particular, se analiza el comportamiento en frecuencia de las condiciones de estabilidad y el comportamiento en el tiempo del sistema en lazo cerrado bajo incertidumbre/variación de la planta.

El resto del artículo se organiza de la siguiente manera: la sección 2 describe el control repetitivo digital, planteando las condiciones de estabilidad y las reglas básicas de diseño. La sección 3 presenta la técnica GPI, describiendo las propiedades de estabilidad y robustez frente a perturbaciones de planta y describiendo su metodología de diseño. En la sección 4 se consolida el diseño del control complementario para el control repetitivo. En la sección 5 se aplica la metodología a un ejemplo lineal realizando las simulaciones y los análisis pertinentes. Finalmente, en la sección 6 , se dan conclusiones y perspectivas de trabajo futuro en este tema de investigación. 


\section{CONTROL REPETITIVO DIGITAL}

El control repetitivo es una técnica basada en el Principio del Modelo Interno (IMP) [18] utilizada para el seguimiento o rechazo de señales periódicas. El IMP establece que para lograr el seguimiento/rechazo asintótico de una señal, el modelo de dicha señal debe ser incorporado en el lazo de control. En el caso de una señal periódica $r(t)$, de periodo $T_{p}$, la obtención del modelo se realiza usando la expansión en series de Fourier de la señal, ver (1)

$r(t)=a_{0}+\sum_{k=1}^{\infty} a_{k} \cos \frac{2 k \pi}{T_{p}}+b_{k} \operatorname{sen} \frac{2 k \pi}{T_{p}} t$.

De esta manera el modelo generador de la señal resulta dado en (2) [19]:

$G_{r}(s)=\frac{1}{s} \prod_{k=1}^{\infty} \frac{\left[\frac{2 k \pi}{T_{p}}\right]^{2}}{s^{2}+\left[\frac{2 k \pi}{T_{p}}\right]^{2}}=\frac{T_{p} e^{-\frac{T_{p} s}{2}}}{1-e^{-T_{p} s}}$.

Con esto, puede observarse que basta simplemente con incluir el siguiente modelo dado en (3) en el lazo de control:

$\bar{G}_{r}(s):=\frac{E_{2}(s)}{E_{1}(s)}=\frac{1}{1-e^{-T p^{s}}}$.

Este modelo cuenta con polos ubicados en $s= \pm j 2 \pi k / T_{p}, k \in \mathbb{N}$ lo que le permite proporcionar ganancia infinita en las frecuencias $2 \pi k / T_{p}, k \in \mathbb{N}$, es decir, en la frecuencia fundamental $1 / T_{p} \mathrm{~Hz}$ y sus armónicos. A su vez, esto asegura un error de seguimiento igual a cero en lazo cerrado cuando este es estable. El modelo (3) puede implementarse usando un bloque de retardo y una realimentación positiva como muestra la Fig. 1.

La implementación discreta de este modelo resulta bastante simple ya que si el periodo $T_{p}$ es un múltiplo entero del periodo de muestreo $T_{s}$, se tiene el controlador mostrado en (4), [20]:

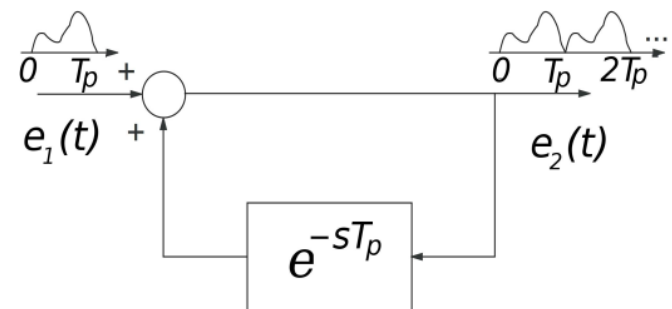

Fig. 1. Modelo interno continuo de una señal periódica. Fuente: Autores

$C_{r}(z)=\frac{1}{z^{N-1}}$,

Con $N=T_{p} / T_{s} \in \mathbb{N}^{+}$. Otros tipos de modelos internos han sido propuestos dependiendo del tipo concreto de señal a tratar [21], [22]. La principal característica de (4) es que tiene $N$ polos uniformemente espaciados sobre el círculo unitario del plano complejo. De esta manera, el modelo proporciona ganancia infinita en las frecuencias $\omega=2 \pi k / N$, con $k=0,1, \ldots, N-1$. Sin embargo, la elevada ganancia del modelo en altas frecuencias compromete la estabilidad y la robustez del sistema. Por ello suelen incluirse dos filtros más: $G_{x}(z)$, usado para asegurar estabilidad y $H(z)$ usado para proveer robustez ante dinámicas no modeladas en las altas frecuencias (ver Fig. 2). De esta manera, el modelo interno resulta de la forma dada en (5),

$G_{i m}(z)=\frac{H(z)}{z^{N}-H(z)}$

Aunque existen diversas estructuras de implementación, la más usada es la estructura "plug-in" mostrada en la Fig. 2. En esta, el controlador repetitivo es adicionado a un controlador existente $G_{c}(z)$, el cual es diseñado para estabilizar la planta $G_{p}(z)$.

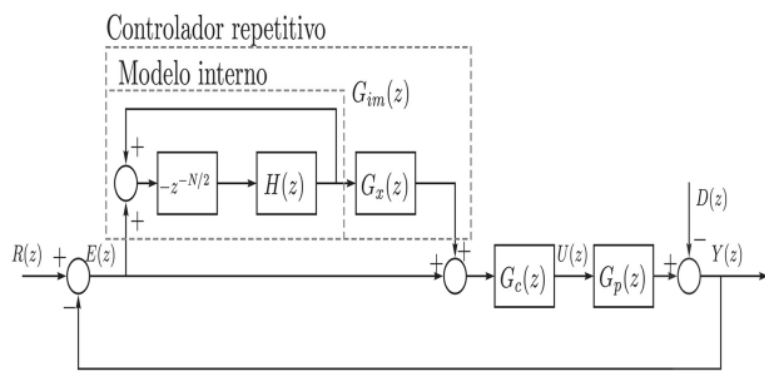

Fig. 2. Estructura plug-in del control repetitivo. Fuente: Autores 
Teorema 1: el lazo cerrado de la Fig. 2 es estable si se cumplen las siguientes dos condiciones [23], [24]. La primera es que el sistema de lazo cerrado sin el controlador repetitivo es estable. Esto es (6) es estable.

$G_{O}(z)=\frac{G_{c}(z) G_{p}(z)}{1+G_{c}(z) G_{p}(z)}$

y la segunda condición es que

$\left\|H(z)\left(1-G_{O}(z) G_{x}(z)\right)\right\|_{\infty}<1$

De (7) se desprenden las siguientes consideraciones de diseño: $H(z)$ se diseña de tal manera que tiene ganancia cercana a 1 en el ancho de banda de interés y proporciona atenuación fuera de él. En general $H(z)$ es un filtro FIR pasa-bajos de fase nula [25]. $G_{x}(z)$ es un filtro a diseñar. Usualmente, en caso de sistemas de fase mínima $G_{x}(z)=k_{r} / G_{o}(z)$ y en caso de sistemas de fase no mínima el diseño sigue el Controlador de Error de Seguimiento de Fase Cero (ZPETC) propuesto en [20].

Cuando la planta presenta incertidumbre y/o variación en sus parámetros a lo largo de la frecuencia de interés puede ser complicado cumplir con la segunda condición. Esto ocasiona que en caso de grandes variaciones o incertidumbre en la planta, el control repetitivo presente problemas de robustez.

A partir de la segunda condición se puede observar que un punto clave es lograr la cancelación de $G_{o}(z)$ [25] mediante el filtro $G_{x}(z)$. Sin embargo, esta cancelación se degrada cuando existen cambios o incertidumbre en la planta. Por lo tanto, es deseable que la cancelación sea lo más robusta posible respecto a cambios o incertidumbre en los parámetros de la planta.

Vale la pena anotar que para los casos en que el sistema es estable y $H(z)=1$, el principio del modelo interno garantiza el desempeño de estado estacionario sin afectarse por la variación de los parámetros de la planta. Para $H(z) \neq 1$ el desempeño sólo podrá afectarse en caso de atenuaciones en la ganancia de la planta de más de $40 \mathrm{~dB}$ en la zona de los armónicos. Por lo tanto no es relevante realizar análisis de desempeño ante los cambio / incertidumbre de los parámetros.

\section{CONTROL ROBUSTO PROPORCIONAL INTEGRAL GENERALIZADO DISCRETO}

La técnica GPI es un procedimiento de diseño de controladores lineales que da lugar a controladores capaces de rechazar diferentes tipos de perturbaciones estructuradas aditivas (i.e. pasos, rampas, parábolas, etc.) [13]. Los controladores GPI se diseñan bajo el contexto del rechazo activo de perturbaciones en el cual se usan modelos simplificados de los sistemas y se estiman o rechazan de manera conjunta $y$ aproximada dinámicas no modeladas, perturbaciones internas y externas del sistema. Los controladores GPI incluyen de manera natural y embebida un modelo polinomial en el tiempo de las perturbaciones dependientes del estado y de aquellas perturbaciones exógenas sin estructura especial alguna.

En este trabajo se realiza una adaptación de la técnica de control GPI para el caso de tiempo discreto. La necesidad de un marco de implementación digital conduce a tomar en consideración adicional tiempos de muestreo y modelos en tiempo discreto. En este contexto, el controlador GPI discreto ha sido menos explorado que su contraparte continua. En [26] se da una formulación teórica del problema de control basada en teoría de módulos. En [27] se propone un control basado en observador GPI discreto para el control de un motor de inducción.

En lo siguiente se asume que se adopta un periodo adecuado de muestreo. El primer aspecto a considerar es el modelo discreto del sistema. Es posible entrar en aproximaciones sofisticadas que generen modelos discretos de gran precisión, pero, por la alta frecuencia de muestreo necesa- 
ria en la implementación y la remarcable robustez del esquema será suficiente con aproximaciones básicas como la dada por la aproximación de Euler, la cual reporta resultados muy satisfactorios [28]. Los errores de discretización serán considerados como parte de la entrada de perturbación. El proceso de discretización puede llevar a posibles ceros inestables, los cuales pueden ser ignorados sin mayores problemas tomando como fundamento los análisis reportados por Goodwin [29] y Hori [30]. Más aún, en el trabajo de Tesfaye y Tomizuka [31] se puede ver un análisis detallado sobre los ceros en sistemas discretizados por el método de Euler lo cual sustenta el empleo de esta metodología.

Considere el siguiente sistema lineal, (8), de una entrada y una salida,

$$
\begin{gathered}
y^{(n)}(t)+a_{n-1} y^{(n-1)}(t)+\cdots+a_{0} y(t) \\
=\kappa u(t) .
\end{gathered}
$$

Donde $y(t)$ es la salida del sistema, $u(t)$ es la entrada de control. El sistema presenta incertidumbre con respecto a los parámetros $\left\{a_{n-1}, a_{n-2}, \ldots, a_{0}, \kappa\right\}$. El sistema es trivialmente diferencialmente plano, con salida plana $y(t)$ [32]. De (8) se define una relación control - salida plana en el dominio del tiempo simplificada (9), sobre la cual se puede incluir un modelo de la incertidumbre de los parámetros compuesto de una perturbación aditiva, $\xi(t)$, y una perturbación multiplicativa, $\kappa$

$y^{(n)}(t)=\kappa u(t)+\xi(t)$,

donde $\xi(t)$ y $\kappa$ son completamente desconocidas pero uniformemente y absolutamente acotadas. Es de anotar que $\xi(t)$ no sólo tiene en cuenta la incertidumbre de los parámetros sino también una parte de la dinámica lineal que se ha omitido en el modelo simplificado.

Para efectos de aplicar una estrategia de control discreta, el primer paso consiste en obtener una discretización del sistema continuo. Como lo habíamos anotado ante- riormente, dada una alta frecuencia de muestreo, se pueden aplicar procesos de discretización simples, en este caso utilizamos la discretización de Euler hacia atrás sobre (9) obteniendo (10),

$$
\sum_{i=0}^{n}(-1)^{i}\left(\begin{array}{c}
n \\
i
\end{array}\right) y_{p}(k-i)=T_{s}^{n} \kappa u(k)+T_{s}^{n} \xi(k)
$$

o bien el dominio Z, se puede expresar como en (11), con $\tilde{\xi}(k)=T_{s}^{n} \xi(k)$ acotada.

$\frac{(z-1)^{n}}{z^{n}} y_{p}(z)=T_{s}^{n} \kappa u(z)+\tilde{\xi}(z)$,

Hacemos la siguiente formulación del problema en tiempo discreto: Dada una trayectoria de referencia $r(k)$ para la salida $y(k)$, proponer una ley de control lineal en tiempo discreto para el sistema (10) de tal forma que se tenga una convergencia, suficientemente cercana de la salida, $y(k)$, hacia la señal de referencia $r(k)$, a pesar de los efectos de la entrada desconocida de perturbación $\tilde{\xi}(k)$. La convergencia aproximada a que se alude implica que el error de seguimiento, $e_{y}(k)=r(k)-y(k)$, converge asintóticamente a una vecindad de cero tan pequeña como se requiera.

En este caso, procedemos a dar la solución en un contexto discreto lineal, donde la señal, $\tilde{\xi}(k)$, se considera como una señal exógena variante en el tiempo. La estrategia de diseño del controlador lineal consiste en aproximar esta señal usando un modelo polinomial interno, realizado por medio de una cadena de $m$ diferencias sucesivas para un entero $m$ suficientemente grande. Es importante anotar que una señal descrita de manera polinómica en $k$ de orden $(m-1)$ es rechazada por medio de un procedimiento de $m$ diferencias sucesivas.

Teorema 2: el control robusto GPI discreto (12),

$$
\begin{aligned}
& u(k)=\frac{1}{\kappa}\left[C_{1}(z)(r(k)-y(k))+C_{2}(z) r(k)\right], \quad \text { (12) } \\
& \text { con } C_{1}(z) \text { y } C_{2}(z) \text { definidos en }(13) \text { y (14), }
\end{aligned}
$$


$C_{1}(z)=\frac{1}{T_{s}^{n}} \frac{\alpha_{n+m-1} z^{n+m-1}+\cdots+\alpha_{1} z+\alpha_{0}}{z^{n}(z-1)^{m}}$

$C_{2}(z)=\frac{1}{T_{s}^{n}} \frac{(z-1)^{n}}{z^{n}}$

lleva el error de seguimiento, $e_{y}(k)=$ $r(k)-y(k)$, asintóticamente y exponencialmente a una vecindad de cero suficientemente pequeña. La vecindad puede ser tan pequeña como se desee de acuerdo con una selección apropiada de los parámetros de ganancia del controlador $\left\{\alpha_{0}, \ldots, \alpha_{n+m-1}\right\}$.

Demostración: la prueba está basada en el hecho que el error de seguimiento $e_{y}(k)$, cumple la ecuación de diferencias lineal perturbada (15), escrita en el dominio Z,

$$
\begin{gathered}
{\left[(z-1)^{n+m}+\alpha_{n+m-1} z^{n+m-1}+\cdots+\alpha_{1} z\right.} \\
\left.+\alpha_{0}\right] e_{y}(z) \\
=T_{s}^{n} z^{n}(z-1)^{m} \tilde{\xi}(z),
\end{gathered}
$$

que puede ser re-escrita como en (16),

$\left[z^{n+m}+l_{n+m-1} z^{n+m-1}+\cdots+l_{1} z+l_{0}\right] e_{y}(z)=$ $T_{s}^{n} z^{n}(z-1)^{m} \tilde{\xi}(z)$,

con

$l_{i}=\left(\begin{array}{c}n+m \\ n+m-i\end{array}\right)+\alpha_{i}$

Los coeficientes $l_{i}$ son combinaciones lineales de los coeficientes $\alpha_{i}$, describiendo una relación biunívoca entre ellos. Por hipótesis, $\tilde{\xi}(k)$, es uniformemente, absolutamente acotada, entonces $(z-1)^{m} \tilde{\xi}(k)$ también es acotado dado que corresponde a una diferencia sucesiva de una serie acotada. Entonces existen coeficientes, $l_{i}$, tales que $e_{y}(k)$ converge a una vecindad pequeña de cero siempre que las raíces del polinomio característico, asociado en la variable compleja $z,\left(z^{n+m}+l_{n+m-1} z^{n+m-1}+\ldots+l_{1} z+\right.$ $l_{0}$ ), estén adecuadamente confinadas dentro del circulo unitario en el plano complejo. En la medida que estas raíces se acercan al origen del plano complejo, más pequeña es la vecindad asociada al error de seguimiento, donde el error de seguimiento $e_{y}(k)$ se mantiene acotado [33].

\section{CONTROL REPETITIVO - GPI}

Con el fin de obtener unas buenas prestaciones para el seguimiento y rechazo de señales periódicas y ser capaces de operar en un contexto de variación de parámetros, se propone el uso combinado del controlador repetitivo y del GPI. La Fig. 3 muestra la arquitectura del controlador propuesto.

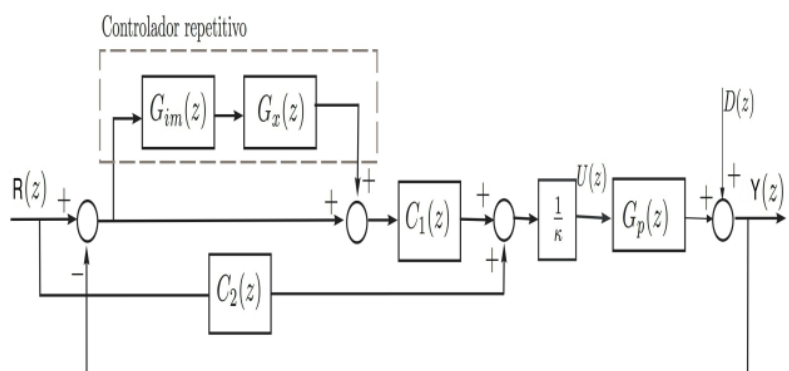

Fig. 3: Diagrama de bloques del controlador GPI-repetitivo propuesto. Fuente: Autores

Dado que el GPI es un controlador lineal, el estudio de la estabilidad del sistema de lazo cerrado puede realizarse aplicando las condiciones descritas en la sección 2. Es decir en primer lugar se diseña $C_{1}(z)$ y $C_{2}(z)$ mediante criterios GPI, se define $G_{c}(z)=C_{1}(z)$ y posteriormente se diseña $G_{x}(z)$ siguiendo el procedimiento de diseño del control repetitivo descrito en la sección 2.

\section{RESULTADOS DE SIMULACIÓN}

Se ha seleccionado como caso de estudio, un caso particular de sistema de segundo orden que considera una gran gama de sistemas prácticos de diferente tipo. Como ejemplos de este tipo de sistema, entre muchos, tenemos: sistema masaresorte-amortiguador, que es un sistema mecánico clásico, utilizado entre muchos fines para el modelado de sistemas de suspensión; motores y servomotores de co- 
rriente directa, ampliamente utilizados para el control de movimiento; circuitos RLC, base fundamental de muchos dispositivos eléctricos; modelos de tanques hidráulicos para el control de nivel.

Los resultados de simulación utilizan la planta de segundo orden de parámetros variantes/inciertos dada en (18):

$G_{p}(s)=\frac{\kappa}{s^{2}+\lambda s+10}$

En la Fig. 4, se muestra la respuesta al paso para diferentes valores de parámetros $\kappa \mathrm{y} \lambda$.

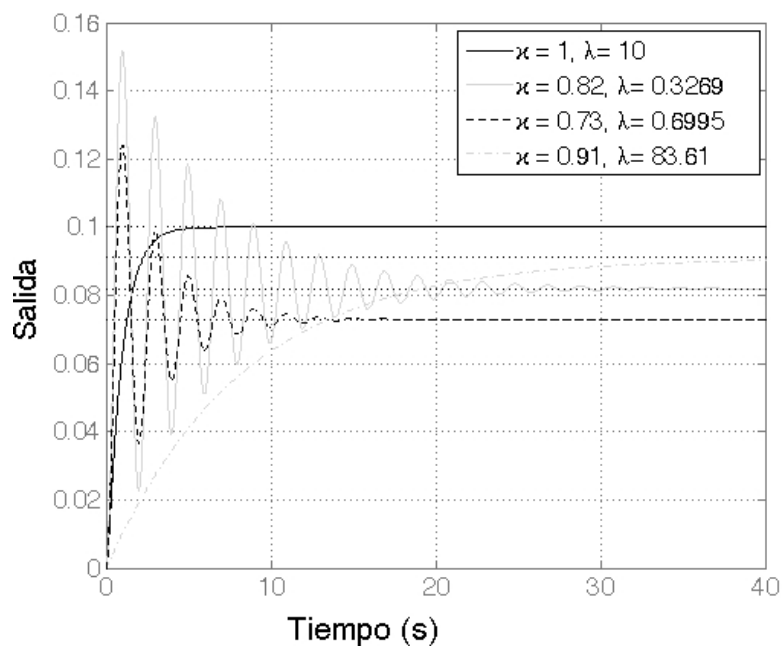

Fig. 4: Respuesta al paso para el sistema de segundo orden con diferentes valores de $\kappa$ y $\lambda$. Fuente: Autores

La estructura del controlador propuesto permite realizar tareas de seguimiento a referencias periódicas o el rechazo a perturbaciones periódicas. Sin pérdida de generalidad, para todos los resultados de simulación presentados, se plantea un problema de seguimiento a una referencia periódica, $r(t)$, dada en (19) (ver Fig. 7).

$$
\begin{gathered}
r(t)=10 \sin \left(\frac{2 \pi t}{T_{p}}\right)+8 \sin \left(\frac{4 \pi t}{T_{p}}+\frac{\pi}{4}\right) \\
-4 \sin \left(\frac{6 \pi t}{T_{p}}+\frac{\pi}{2}\right),
\end{gathered}
$$

con $T_{p}=1$. Los parámetros $\kappa$ y $\lambda$ son susceptibles de variación/incertidumbre, siendo $k=1$ y $\lambda=10$ los valores nominales.
El diseño del controlador GPI sigue lo expuesto en la sección 3 con la aproximación a un sistema de segundo orden $(n=2)$, dada en (20),

$\frac{(z-1)^{2}}{z^{2}} y_{p}(z)=T_{s}^{2} \kappa u(z)+\tilde{\xi}(z)$.

Con esto y definiendo el grado de robustez como $m=3$, el controlador resulta dado en (21) y (22),

$C_{1}(z)$
$=\frac{4268 z^{4}-17007 z^{3}+25414 z^{2}+16878 z+4203}{z^{2}(z-1)^{3}}$

$C_{2}(z)=\frac{1}{T_{S}^{2}} \frac{(z-1)^{2}}{z^{2}}$

El control repetitivo es diseñado para la planta nominal (17) discretizada a un periodo de muestreo de $T_{s}=5 \mathrm{~ms}$. El periodo de la señal a seguir/rechazar se define como $T_{p}=1 \mathrm{~s}$ que corresponde con la frecuencia fundamental de $f n=1 \mathrm{~Hz}$. Con esto $N=T_{p} / T_{s}=200$. El controlador $G_{c}(z)$ se calcula como $G_{c}(z)=C_{1}(z)$. Adicionalmente, se define $H(z)=0,25 z+0,5+$ $0,25 z^{-1}$ y $k_{r}=0,3$, y siguiendo el procedimiento habitual en el control repetitivo $G_{x}(z)=1 / G_{O}(z)$.

Con la finalidad de comparar la robustez de diferentes diseños, se han considerado: un control proporcional $\mathrm{P}$, un control proporcional integral PI y una red de adelanto RA, todos ellos diseñados para obtener el mismo ancho de banda que el obtenido con el controlador GPI y con los mismos valores para $N, H(z), k_{r}$ e igual procedimiento de diseño para $G_{x}(z)$. Los controladores obtenidos son: $C_{P}(z)=100, C_{P I}(z)=$ $(100 z-99,9) /(z-1)$ y $C_{R A}(z)=(268,5 z-$ $259,4) /(z-0,8854)$ para el control P, PI y RA respectivamente. Estos controladores se sintonizaron para obtener el mismo desempeño en las condiciones nominales de la planta (ver Fig. 7). Se han escogido estos controladores ya que comúnmente son empleados como controladores existentes 
en el diseño de controladores repetitivos [4], [34]-[37].

El primer análisis de robustez se realiza sobre las condiciones de estabilidad presentadas en el Teorema 1. Se construyen 20 diferentes modelos de la planta con los parámetros $\kappa$ y $\lambda$ definidos en los intervalos $[1,1 / 6]$ y $[0,100]$ respectivamente (esto se puede realizar fácilmente empleando el toolbox de control robusto de Matlab, con los comandos ureal para la definición de los intervalos y el comando usample para la obtención de los 20 modelos de muestra). Con esto se realiza el cálculo de $G_{O}(z)$ para cada uno de los controladores y se procede a verificar las condiciones de estabilidad.

La Fig. 5 muestra el diagrama de Nyquist de $G_{p}(z) G_{c}(z)$ para verificar la condición 1 de los cuatro controladores $\mathrm{P}$, PI, RA y GPI para los 20 diferentes casos de la planta. Como puede verse, solo en un caso el controlador PI resulta inestable al presentarse el encerramiento del punto (1,0). Con esto, sólo este caso debería ser eliminado para continuar con el análisis de la segunda condición. Todos los casos para los controladores P, RA, y GPI resultan estables.

El segundo criterio de estabilidad usado para comparar los cuatro controladores diseñados está basado en la segunda condición de estabilidad del Teorema 1. Para verificar esta condición se grafica la respuesta en magnitud de $H(z)(1-G o(z) G x(z))$ con respecto a la frecuencia, cuyo máximo corresponde con el valor de la norma infinito.

La Fig. 6 muestra la respuesta en magnitud de $H(z)(1-G o(z) G x(z))$ para los cuatro controladores propuestos y los 20 diferentes modelos de la planta de acuerdo con los posibles valores de $\kappa$ y $\lambda$ dentro de los intervalos mencionados anteriormente. Se puede observar que para los controladores P y PI muchos casos no cumplen la condición de estabilidad, mientras que para el controlador RA solo algunos casos no cumplen esta condición.

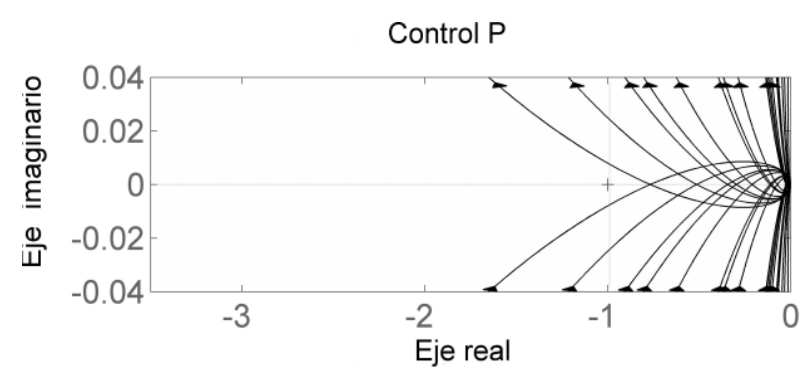

a)

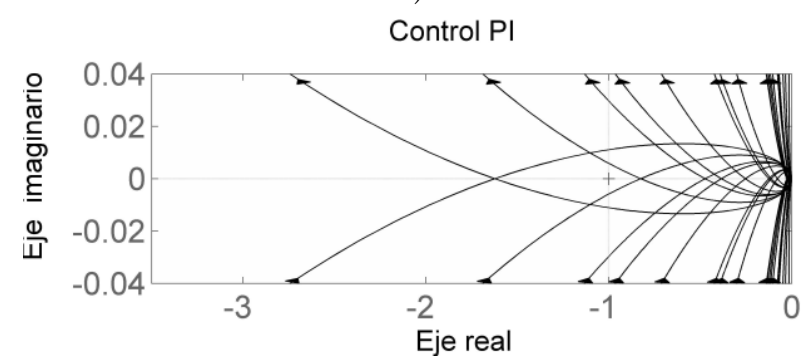

b)

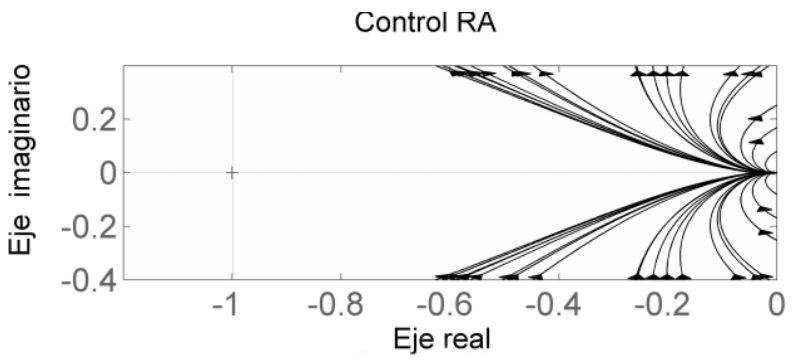

c)

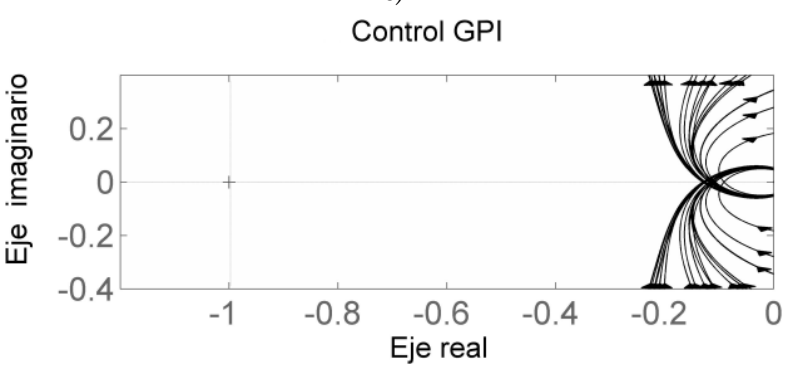

d)

Fig. 5. Diagrama de Nyquist para los controladores: a) P, b) PI, c) RA y d) GPI con 20 diferentes modelos de la planta. Fuente: Autores

Por otro lado, para el control GPI todos los casos cumplen con la condición de estabilidad. Esto muestra como el control GPI logra un grado de robustez mayor ante la incertidumbre en los parámetros comparado con estos controladores. 


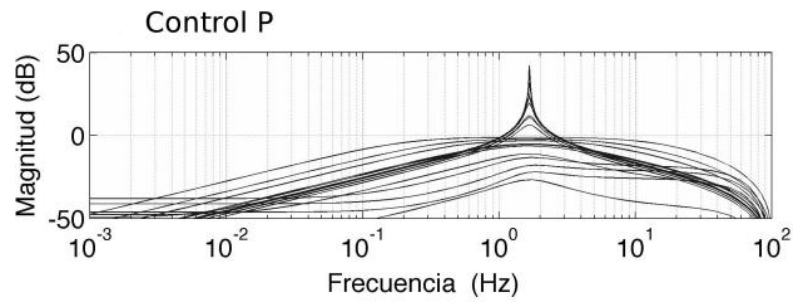

a)

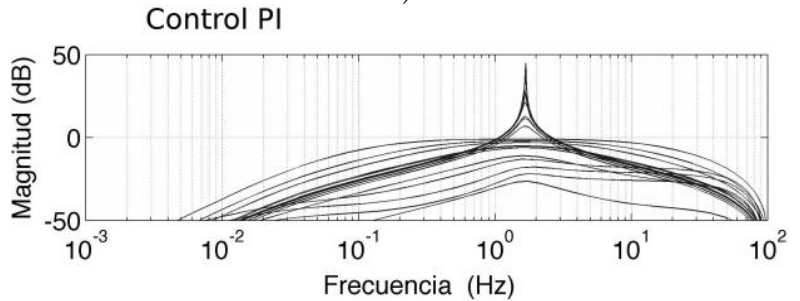

b)

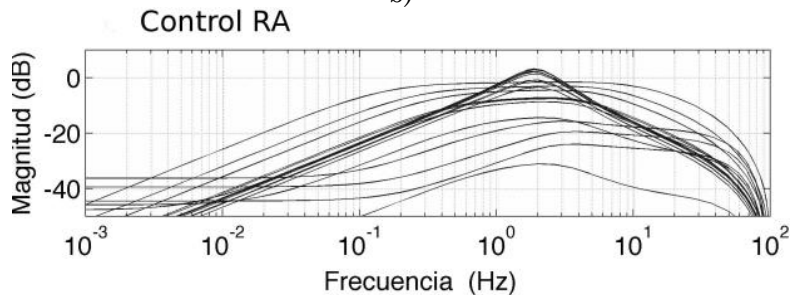

c)

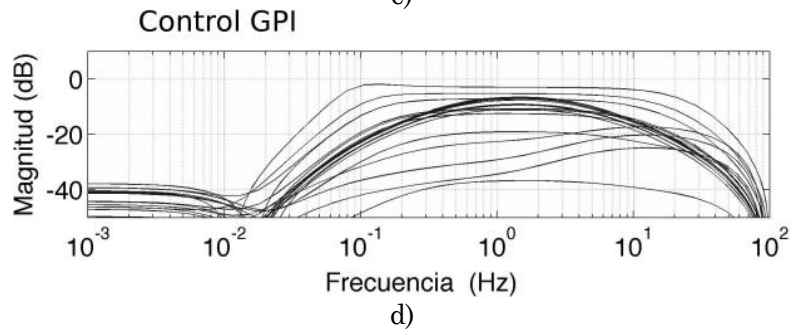

Fig. 6. Respuesta en frecuencia de $\left.\mathrm{H}(\mathrm{z})\left(1-\mathrm{Go}_{\mathrm{o}} \mathrm{z}\right) \mathrm{Gx}(\mathrm{z})\right)$ para los controladores a) P, b) PI, c) RA, d) GPI con 20 diferentes modelos de la planta. Fuente: Autores

Para la siguiente simulación se utiliza una variación en el tiempo tipo rampa de los parámetros $\kappa$ y $\lambda$ en los intervalos $\kappa \in$ $[1,1 / 6], \lambda \in[0,100]$. De esta manera, $\lambda$ cambia de su valor nominal 10 a 0 en $t=$ $100 \mathrm{~s}$ a una tasa de -4 unidades/s y el parámetro $\kappa$ cambia de 1 a $1 / 6$ en $t=150 \mathrm{~s}$ a una tasa de $-0,3$ unidades/s. La Fig. 7 muestra el error de seguimiento la señal de referencia y la salida en el tiempo obtenido por el controlador P, PI, RA y GPI ante esta variación de parámetros.

Como puede verse, los diseños P y PI se desestabilizan debido al cambio en $\lambda$ en $t=$ 100 s. El diseño RA soporta el cambio en $\lambda$ pero no el subsiguiente cambio de $\kappa$ en $t=$ $150 \mathrm{~s}$, después de lo cual se desestabiliza.

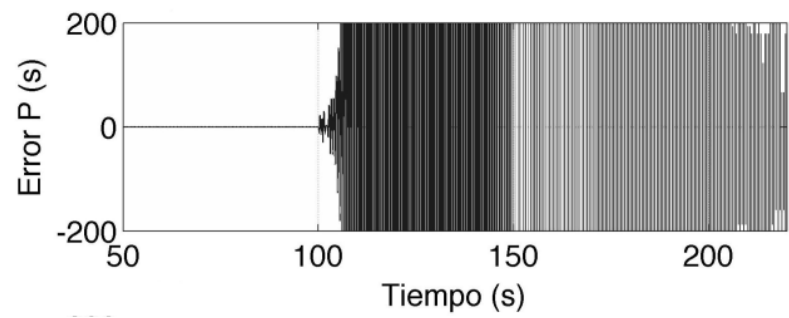

a)

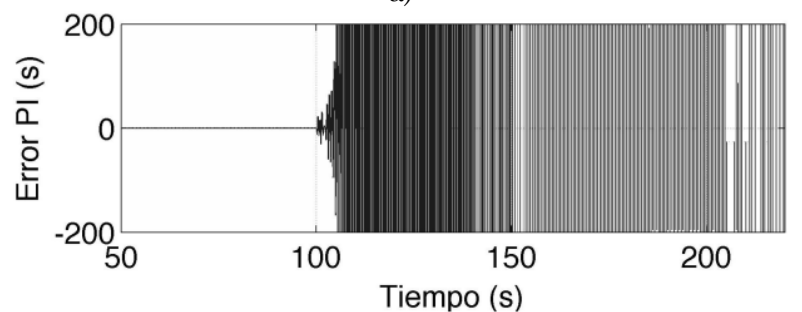

b)

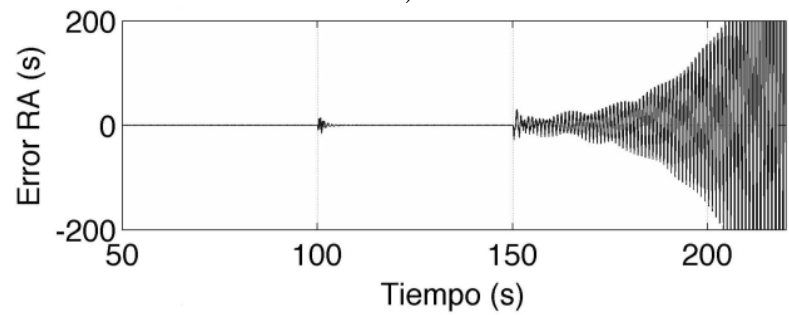

c)

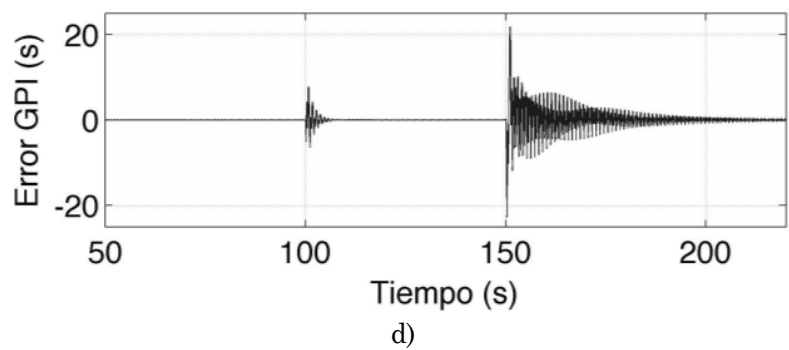

Fig. 7. Respuesta en el tiempo obtenido para el controlador a) P, b) PI, c) RA y d) GPI ante variaciones en el tiempo de los parámetros $\kappa$ y $\lambda$. Derecha: error de seguimiento, izquierda: referencia y salida. Fuente: Autores

El control GPI por el contrario, logra ser robusto ante los cambios en $\kappa$ y $\lambda$ manteniendo a la vez un error de seguimiento pequeño. Vale la pena mencionar que para los valores $\kappa=1 / 6$ y $\lambda=0,5$ el lazo cerrado $G o(z)$ de los cuatro controladores resulta estable, sin embargo, con estos mismos valores la segunda condición del Teorema 1 sólo se cumple en el caso del control GPI. 


\section{CONCLUSIONES}

La técnica de control repetitivo fue exitosamente combinada con la técnica de control GPI para proveer una adecuada robustez del sistema frente a incertidumbre/variación paramétrica. Se presentó una metodología de diseño en tiempo discreto del control repetitivo en complemento con el control GPI para sistemas lineales con incertidumbre. Se establecen condiciones de estabilidad para el diseño repetitivoGPI.

Las comparaciones de las condiciones de estabilidad demuestran un mayor grado de robustez del controlador GPI con respecto a los otros controles clásicos utilizados en la comparación. Los resultados satisfactorios de este estudio particular animan a continuar con análisis más generales que incluyan control repetitivo para sistemas no lineales y sistemas con frecuencia variable de las señales periódicas a seguir/rechazar.

\section{REFERENCIAS}

[1] S.-C. Wu and M. Tomizuka, "An Iterative Learning Control design for Self-ServoWriting in Hard Disk Drives," Mechatronics, vol. 20, no. 1, pp. 53-58, Feb. 2010.

[2] A. Tayebi, S. Abdul, M. B. Zaremba, and Y. Ye, "Robust Iterative Learning Control Design: Application to a Robot Manipulator," IEEE/ASME Trans. Mechatronics, vol. 13, no. 5, pp. 608-613, Oct. 2008.

[3] D. H. Kim and T.-C. Tsao, "Robust performance control of electrohydraulic actuators for electronic cam motion generation," IEEE Trans. Control Syst. Technol., vol. 8, no. 2, pp. 220-227, Mar. 2000.

[4] J. D. Álvarez, L. J. Yebra, and M. Berenguel, "Repetitive control of tubular heat exchangers," J. Process Control, vol. 17, no. 9, pp. 689-701, Oct. 2007.

[5] K. Zhou, D. Wang, and G. Xu, "Repetitive controlled three-phase reversible PWM rectifier," in Proceedings of the 2000 American Control Conference. ACC (IEEE Cat. No.00CH36334), 2000, pp. 125-129.

[6] R. Grino, R. Cardoner, R. Costa-Castello, and E. Fossas, "Digital Repetitive Control of a Three-
Phase Four-Wire Shunt Active Filter," IEEE Trans. Ind. Electron., vol. 54, no. 3, pp. 14951503, Jun. 2007.

[7] S. S. Ge and I. M. Y. Mareels, "Adaptive repetitive learning control of robotic manipulators without the requirement for initial repositioning," IEEE Trans. Robot., vol. 22, no. 3, pp. 563-568, Jun. 2006.

[8] W. Chen and Z. Zhang, "Nonlinear adaptive learning control for unknown time-varying parameters and unknown time-varying delays," Asian J. Control, vol. 13, no. 6, pp. 903-913, Nov. 2011.

[9] M. Wu, L. Zhou, J. She, and Y. He, "Design of robust output-feedback repetitive controller for class of linear systems with uncertainties," Sci. China Inf. Sci., vol. 53, no. 5, pp. 1006-1015, May 2010.

[10] L. Yun, T. Xu, and Y. Sun, "Repetitive learning control for nonlinear systems with both parametric and non-parametric uncertainties systems," Control Decis., vol. 25, no. 12, pp. 1880-1884, 2010.

[11] Y. Shi, R. W. Longman, and M. Q. Phan, "An algorithm for robustification of repetitive control to parameter uncertainties," Adv. Astronaut. Sci., vol. 136, pp. 1953-1966, 2010.

[12]Z. Gao, "Active disturbance rejection control: a paradigm shift in feedback control system design,” in 2006 American Control Conference, 2006, pp. 1-7.

[13] H. Sira-Ramirez, A. Luviano-Juárez, and J. Cortés-Romero, "Control lineal robusto de sistemas no lineales diferencialmente planos," Rev. Iberoam. Automática e Informática Ind. RIAI, vol. 8, no. 1, pp. 14-28, Jan. 2011.

[14] H. Sira-Ramírez, C. López-Uribe, and M. Velasco-Villa, "Linear Observer-Based Active Disturbance Rejection Control of the Omnidirectional Mobile Robot," Asian J. Control, vol. 15, no. 1, pp. 51-63, Jan. 2013.

[15]H. Sira-Ramírez, A. Luviano-Juárez, and J. Cortés-Romero, "Flatness-based linear output feedback control for disturbance rejection and tracking tasks on a Chua's circuit," Int. J. Control, vol. 85, no. 5, pp. 594-602, May 2012.

[16] H. Sira-Ramirez, F. Gonzalez-Montanez, J. A. Cortes-Romero, and A. Luviano-Juarez, "A Robust Linear Field-Oriented Voltage Control for the Induction Motor: Experimental Results," IEEE Trans. Ind. Electron., vol. 60, no. 8, pp. 3025-3033, Aug. 2013.

[17]H. Sira-Ramírez, A. Luviano-Juárez, and J. Cortés-Romero, "Robust input-output sliding mode control of the buck converter," Control Eng. Pract., vol. 21, no. 5, pp. 671-678, May 2013.

[18] B. A. Francis and W. M. Wonham, "The internal model principle of control theory," Automatica, vol. 12 , no. 5, pp. 457-465, Sep. 1976. 
[19] Y. Yamamoto, "Learning Control and Related Problems in Infinite-Dimensional Systems," in Essays on Control, vol. 14, H. L. Trentelman and J. C. Willems, Eds. Birkhauser, 1993, pp. 191222.

[20]M. Tomizuka, T.-C. Tsao, and K. Chew, "Analysis and Synthesis of Discrete-Time Repetitive Controllers," J. Dyn. Syst. Meas. Control, vol. 111, no. 3, p. 353, 1989.

[21]R. Griñó and R. Costa-Castelló, "Digital repetitive plug-in controller for odd-harmonic periodic references and disturbances," Automatica, vol. 41, no. 1, pp. 153-157, Jan. 2005.

[22] G. Escobar, P. G. Hernandez-Briones, R. E. Torres-Olguin, A. A. Valdez, and M. HernndezGomeza, "A repetitive-based controller for the compensation of $6 \mathrm{l} \pm 1$ harmonic components," in 2007 IEEE International Symposium on Industrial Electronics, 2007, pp. 3397-3402.

[23] T. Inoue, M. Nakano, T. Kubo, S. Matsumoto, and H. Baba, "High Accuracy Control of a Proton Synchroton Magnet Power Supply," in Proceedings of the 8th IFAC World Congress, 1981, pp. 216-220.

[24] R. Costa-Castello, J. Nebot, and R. Grino, "Demonstration of the Internal Model Principle by Digital Repetitive Control of an Educational Laboratory Plant," IEEE Trans. Educ., vol. 48, no. 1, pp. 73-80, Feb. 2005.

[25] T. Inoue, "Practical repetitive control system design," in 29th IEEE Conference on Decision and Control, 1990, pp. 1673-1678 vol.3.

[26] M. Fliess and R. Marquez, "Une approche intrinsèque de la commande prédictive linéaire discrète," J. Eur. Syst. Autom., vol. 35, pp. 127 $147,2001$.

[27]J. Cortes-Romero, A. Luviano-Juarez, and H. Sira-Ramirez, "A discrete-time observer-based control for induction motors," in IECON 2010 36th Annual Conference on IEEE Industrial Electronics Society, 2010, pp. 17-22.

[28] A. Elfadili, F. Giri, H. Ouadi, and L. Dugard, "Discrete-time modelling of induction motors with consideration of magnetic saturation," in IECON 2006 - 32nd Annual Conference on IEEE Industrial Electronics, 2006, pp. 5119-5124.

[29] G. C. Goodwin, R. L. Leal, D. Q. Mayne, and R. H. Middleton, "Rapprochement between continuous and discrete model reference adaptive control," Automatica, vol. 22, no. 2, pp. 199-207, Mar. 1986.

[30] N. Hori, D. Bitner, P. Nikiforuk, and P. Ukrainetz, "Robust adaptive control of electrohydraulic servo systems using the Euler operator," in International Conference of Control, Edinburgh, 1991, pp. 671-676.

[31]A. Tesfaye and H. Tomizuka, "Zeros of discretized continuous systems expressed in the Euler operator-an asymptotic analysis," IEEE Trans. Automat. Contr., vol. 40, no. 4, pp. 743747, Apr. 1995.

[32] H. Sira-Ramirez and S. K. Agrawal, Diferencially Flat Systems. CRC Press, 2004, p. 450.

[33] T. Kailath, Linear Systems. Prentice Hall, 1980, p. 682.

[34] S. Doki and M. Ishida, "Suppression of harmonic current in vector control for IPMSM by utilizing repetitive control," in 2002 IEEE International Conference on Industrial Technology, 2002. IEEE ICIT' '02., 2002, pp. 264-267.

[35] X. H. Wu, S. K. Panda, and J.-X. Xu, "Design of a Plug-In Repetitive Control Scheme for Eliminating Supply-Side Current Harmonics of Three-Phase PWM Boost Rectifiers Under Generalized Supply Voltage Conditions," IEEE Trans. Power Electron., vol. 25, no. 7, pp. 18001810, Jul. 2010.

[36] W.-S. Yau and M.-C. Tsai, "Repetitive control design for linear servo systems," in Proceedings of the 1999 American Control Conference (Cat. No. 99CH36251), 1999, vol. 5, pp. 3728-3732.

[37]R. Costa-Castelló, S. Malo, and R. Griñó, "High Performance Repetitive Control of an Active Filter under Varying Network Frequency," in Proceedings of the 17th IFAC World Congress, 2008, pp. 3344-3349. 